\title{
Central line-associated bloodstream infections in neonates
}

Hye Jung Cho, MD, Hye-Kyung Cho, MD, PhD

Department of Pediatrics, Gil Medical Center, Gachon University College of Medicine, Incheon, Korea

Newborn infants, including premature infants, are high-risk patients susceptible to various microorganisms. Catheter-related bloodstream infections are the most common type of nosocomial infections in this population. Regular education and training of medical staffs are most important as a preventive strategy for central line-associated bloodstream infections (CLABSIs). Bundle approaches and the use of checklists during the insertion and maintenance of central catheters are effective measures to reduce the incidence of CLABSIs. Chlorhexidine, commonly used as a skin disinfectant before catheter insertion and dressing replacement, is not approved for infants $<2$ months of age, but is usually used in many neonatal intensive care units due to the lack of alternatives. Chlorhexidine-impregnated dressing and bathing, recommended for adults, cannot be applied to newborns. Appropriate replacement intervals for dressing and administration sets are similar to those recommended for adults. Umbilical catheters should not be used longer than 5 days for the umbilical arterial catheter and 14 days for the umbilical venous catheter. It is most important to regularly educate, train and give feedback to the medical staffs about the various preventive measures required at each stage from before insertion to removal of the catheter. Continuous efforts are needed to develop effective and safe infection control strategies for neonates and young infants.

Key words: Central venous catheter, Intensive care units, Bacteremia, Newborn infant

\section{Introduction}

Healthcare-associated infections (HAIs) in the neonatal intensive care unit (NICU) result in significant morbidity and late mortality among hospitalized newborns. Almost 25\% of very low birth weight (VLBW, $<1,500$-g birthweight) infants experience more than one episode of nosocomial infection, which is a major threat to the infants. The risk factors for HAIs in the NICU include prematurity, low birthweight, invasive procedures, indwelling vascular catheters, endotracheal tubes, ventricular shunts, parenteral nutrition with lipid emulsions, alterations in the skin and mucous membrane barriers, broad-spectrum antibiotics, and prolonged hospitalization. ${ }^{1)}$

Bloodstream infections (BSIs) are the most common type of HAI occurring in the NICU. Late-onset sepsis occurring after 3 days of birth occurs in 20\%-36\% of VLBW babies. ${ }^{1)}$ Most cases of late-onset sepsis are caused by central venous catheters (CVCs). Premature infants who experience catheter-related infections have a high mortality rate, poor growth and neurodevelopmental outcomes, as well as prolonged hospital stay, leading to increased medical costs. ${ }^{2)}$ Therefore, more efforts are needed to reduce the incidence of CVC-related infections among NICU patients, and to enhance their survival and prognosis.

Here, we will focus on the basic concepts of catheter-related BSIs in NICUs and briefly review strategies for preventing them.
Corresponding author: Hye-Kyung Cho, MD, PhD Department of Pediatrics, Gil Medical Center, Gachon University College of Medicine, 21, Namdongdae-ro 774 beongil, Namdong-gu, Incheon 21565, Korea

Tel: +82-32-458-2652

Fax: +82-32-460-2362

E-mail: hkcho@gilhospital.com

https://orcid.org/0000-0003-0990-1350

Received: 2 September, 2018

Revised: 6 December, 2018

Accepted: 19 December, 2018

Copyright (C) 2019 by The Korean Pediatric Society

This is an open-access article distributed under the terms of the Creative Commons Attribution NonCommercial License (http://creativecommons.org/ licenses/by-nc/4.0/ which permits unrestricted noncommercial use, distribution, and reproduction in any medium, provided the original work is properly cited. 


\section{Definition of catheter-related BSIs}

There are 2 major definitions of BSIs associated with CVCs: catheter-related bloodstream infection (CRBSI) and central line-associated bloodstream infection (CLABSI) (Table 1). ${ }^{3)}$ SI refers to the presence of bacteremia originating from the intravenous (IV) catheter and is more of a clinical definition. To diagnose this, a specific laboratory test is required to confirm that the source of the BSI is a catheter. CLABSI, on the other hand, refers to a primary BSI that has not been associated with infection at other sites in a patient who had a central line within 48 hours of symptom onset. Culturing the catheter tip or peripheral blood is not a criterion for CLABSI. The CLABSI definition is more practical than the CRBSI definition for surveillance. However, it may overestimate the true rate of CVC-related infections.

\section{Need for the definition of BSI specifically adapted for neonates}

According to the National Healthcare Safety Network in the United States, CLABSI in children $\leq 1$ year of age is defined as primary BSI with symptoms of fever $\left(>38^{\circ} \mathrm{C}\right)$, hypothermia $\left(<36^{\circ} \mathrm{C}\right)$, apnea, or bradycardia. BSIs caused by a recognized pathogen (e.g., Escherichia coli) isolated from $\geq 1$ blood culture or common skin commensal (e.g., coagulase-negative staphylococci [CoNS]) cultured from $\geq 2$ blood cultures drawn on separate occasions are included. ${ }^{4)}$ However, these definitions for children aged $\leq 1$ year are not suitable in some aspects for neonates due to differences in the symptoms of infection. Body temperature seldom exceeds $38.0^{\circ} \mathrm{C}$, artificially adjusted by lowering the incubator temperature, but hypothermia, hyperthermia and temperature instability can occur. Other definitions often include temperature instability, tachycardia, prolonged recapillarization, metabolic acidosis, and newly developed hyperglycemia. ${ }^{5)}$ Some attempts have been made to include other diagnostic methods such as antigen testing or to include both 'laboratory confirmed' BSIs and clinical BSIs. ${ }^{6}$

\section{Etiology of CLABSI in NICUs}

In the NICU population, the etiological organism of CLABSI is similar to that among adults, but the proportion of gram-negative organisms such as Klebsiella pneumoniae, E. coli, and Enterobacter cloacae is relatively high. This is due to increased bacterial translocation from the gastrointestinal tract, especially in babies receiving long-term parenteral nutrition, which is associated with impaired bowel wall function and primary mucosal pathology, gastrointestinal surgery or lack of enteral nutrition. ${ }^{7)}$ While gram-positive bacteria including CoNS and Staphylococcus aureus are more important in developed countries, gram-negative bacteria are found more often in developing countries. ${ }^{7-9)}$ In Korea, the proportion of gram-positive bacteria has been reported to be higher. ${ }^{10)}$

\section{Pathogenesis of CLABSI}

There are several potential pathways and sources causing CLABSI (Fig. 1). ${ }^{11)}$ First, the catheter insertion site can serve as a port of entry

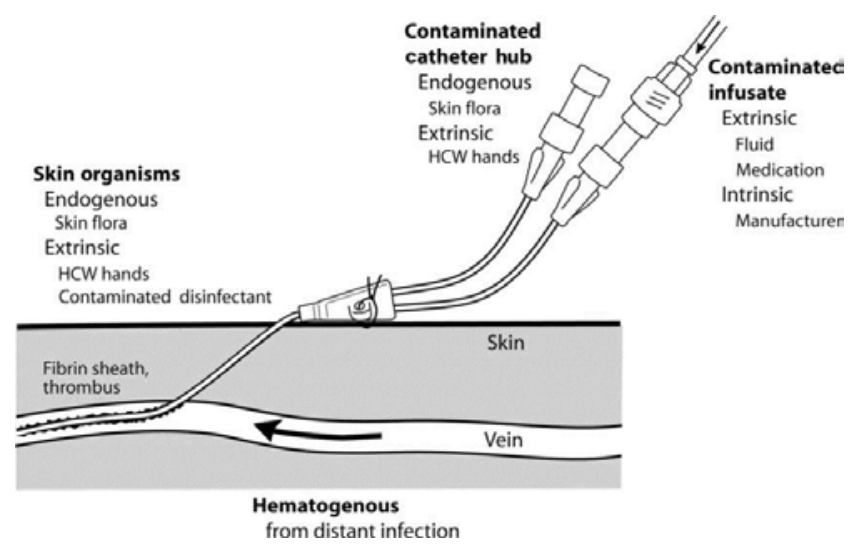

Fig. 1. Potential pathways and sources leading to central line-associated bloodstream infections. HCW, health care worker. Reprinted from Crnich et al. Clin Infect Dis 2002;34:1232-42, with permission of Oxford University Press. $^{11)}$

Table 1. Two definitions of central venous catheter-related bloodstream infections

\begin{tabular}{|c|c|}
\hline Bloodstream infection & Definitions \\
\hline \multirow[t]{4}{*}{ Catheter-related bloodstream infection } & $\begin{array}{l}\text { Clinical signs of sepsis and positive peripheral blood culture in the absence of an obvious source other than CVC } \\
\text { with one of the following: }\end{array}$ \\
\hline & $\begin{array}{l}\text { Positive semiquantitative ( }>15 \mathrm{CFU} \text { ) or quantitative (>103 CFU) culture from a part of the catheter with the same } \\
\text { organisms isolated peripherally }\end{array}$ \\
\hline & Simultaneous quantitative blood cultures with a ratio of $\geq 3: 1$ (CVC vs. peripheral) \\
\hline & Time difference of $\leq 2$ hours leading to culture positive between CVC and peripheral cultures \\
\hline \multirow[t]{2}{*}{ Central line-associated bloodstream infection } & $\begin{array}{l}\text { Primary bloodstream infection in a patient who had a central line within the } 48 \text { hours period before development } \\
\text { of infection }\end{array}$ \\
\hline & Infection must not be related to an alternative cause \\
\hline
\end{tabular}

CVC, central venous catheter; CFU, colony forming unit.

Adapted from Bell T, et al. Infect Dis Clin North Am 2017;31:551-9, with permission of Elsevier. ${ }^{3)}$ 
for organisms. In this case, endogenous skin flora of the patient and extrinsic organisms from the hands of healthcare workers or even from contaminated disinfectants are potential sources. Second, during catheter hub operation it can be contaminated with organisms from the patient's skin flora or healthcare workers' hands. Third, fluids or drugs can be contaminated during the preparation process or the manufacturing process of the company. Finally, there may be a secondary infection that causes catheter infection due to hematogenous dissemination of infection in other parts of the body. ${ }^{11}$

One of the major steps in the process of catheter infection is the formation of biofilms extraluminally and intraluminally. Biofilm is composed of bacteria embedded within an extracellular polysaccharide matrix on the catheter surface. ${ }^{12)}$ These biofilms develop within 24 hours of catheter insertion by organisms on the skin and are mainly formed on the external surface of the catheter. Over time, a biofilm is formed on the inner surface of the catheter, which occurs mainly in the process of connecting and disconnecting the catheter hub. This usually occurs in long-term catheters that remain in situ for more than 10 days. Bacteria embedded in biofilms are less susceptible to the effects of antibiotics due to their low metabolic rate. The minimum inhibitory concentrations for microorganisms in a biofilm are known to be up to 1,000 fold higher than planktonic organisms. ${ }^{13)}$ In addition, biofilms are highly impenetrable to antibiotics. Therefore, it is difficult to eradicate the infection without removing the infected device.

\section{Prevention strategies for CLABSI in the NICU}

\section{Education, training, and staffing}

It is very important to educate medical staff regularly and repeatedly about the indications for IV catheter use, the appropriate practices during catheter insertion and maintenance, and other policies for catheter-related infection prevention. This involves encouraging them to practice what they already know by regularly reminding, rather than giving them new principles. ${ }^{14)}$ It is also necessary to regularly assess how well all staffs involved in the insertion and maintenance of IV catheters are aware of the guidelines and how well they are practicing them. In addition, an effective way to prevent CLABSI is by allowing only skilled medical personnel to manage insertion and maintenance of peripheral and central intravascular catheters, for example, designated nurse peripherally inserted central catheter (PICC) teams (i.e., specially trained nurses who are responsible for PICC insertions at a hospital). ${ }^{15)}$ It should also be ensured that appropriate patient-to-nurse ratios are maintained within the NICU, as elevated patient-to-nurse ratios are associated with increased CLABSI incidence. ${ }^{16)}$

\section{Selection of the PICC site}

There is no preferred location of the upper or lower limb as a PICC location. ${ }^{15,17,18)}$ Further research is needed on this issue. For longterm surgically implanted central lines, the subclavian or femoral vein is preferred to the internal jugular vein due to the high risk of CLABSI. ${ }^{19,20)}$

\section{Intervention bundle for CVC insertion and maintenance}

To prevent CLABSI, an intervention bundle is recommended for insertion and maintenance of CVC (Table 2). ${ }^{21)}$ When using an intervention bundle, it is better to use a checklist to encourage compliance with medical staff training and recommendations. In a United States study, the incidence of CLABSI was reduced by about 40\% after the administration of a bundle compared to before. In addition, there was a difference in the incidence of CLABSIs depending on the utilization rate of the checklist, which suggested that using the checklist as well as the bundle could prevent more cases of CLABSI. ${ }^{8)}$

Table 2. Suggestive elements of the bundles for insertion and maintenance of central catheters to prevent catheter-related infection (elements of the bundle can be adjusted according to the environment of each neonatal intensive care unit)

Insertion bundle Maintenance bundle

Establish a central catheter kit or cart with all the items required for the procedure

Perform hand hygiene with an alcohol-based product or disinfectantcontaining soap before and after palpating insertion sites and before and after inserting the central catheter

Use maximal barrier precautions (sterile gown, sterile gloves, surgical mask, hat, and large sterile drape)

Disinfect the skin with a proper antiseptic (e.g., 2\% chlorhexidine, $70 \%$ alcohol) before catheter insertion

Use either a sterile transparent semipermeable dressing or sterile gauze to cover the insertion site
Perform hand hygiene with an alcohol-based product or disinfectant containing soap before or after accessing the catheter, or before or after changing the dressing.

Daily access the catheter insertion sites to identify signs of infection and dressing integrity

At least, if the dressing is damp, soiled or loosened, change the dressing aseptically and disinfect the skin around the insertion site with a suitable disinfectant (e.g., $2 \%$ chlorhexidine, $70 \%$ alcohol).

Develop and use standardized intravenous tubing setup and changes

Maintain aseptic technique and scrub the hub using appropriate disinfectant when replacing intravenous tubing and when accessing the catheter

Daily review catheter necessity to immediately eliminate when it is no longer essential

Adapted from Schulman J, et al. J Perinatol 2009;29:591-9, with permission of Springer Nature Publishing AG. ${ }^{21)}$ 


\section{Skin antiseptics in infants and neonates}

Before inserting a peripheral venous catheter, it is recommended to disinfect the skin using $70 \%$ alcohol, tincture of iodine or alcoholic chlorhexidine gluconate (CHG) solution. Before inserting the CVC or peripheral arterial catheter and changing the dressing, it is necessary to disinfect the skin with $>0.5 \%$ CHG with alcohol, tincture of iodine, iodophor, or 70\% alcohol. ${ }^{14)}$ There is a lack of studies comparing CHG formulations with alcohol or povidoneiodine with alcohol preparations. The disinfectant must be dried before inserting the catheter.

No recommendation is made about the preferred antiseptic for catheter insertion and exit site care. ${ }^{22)}$ The use of CHG is not approved in infants $<2$ months of age due to reports of serious contact dermatitis as well as concerns about systemic absorption and possible toxic effects. Nevertheless, many centers currently use it. In a survey on the use of CHG in NICUs in the US, more than half reported having used CHG. Of these, 51\% experienced adverse skin reactions, most of which occurred in VLBW infants. No respondents experienced neurologic toxicities in this study.

Due to the risk of thyroid dysfunction associated with the use of iodine agents, it has not been widely used in NICUs. ${ }^{23-26)}$

\section{In-line filters}

There are 2 main IV filters according to pore size; the $0.22-\mu$ filter is used for aqueous solutions, and the $1.2-\mu$ filter is recommended for larger molecule solutions such as lipids. The $0.22-\mu$ filter has also been reported to remove air, microorganisms and particulate matter. However, in-line filters are not recommended for use only to prevent CLABSI. A meta-analysis of in-line filter effects on mortality and morbidity in neonates reported no difference in death, proven septicemia, or suspected septicemia during admission. ${ }^{27)}$

\section{Catheter dressing regimens}

Gauze dressing and transparent-semipermeable dressing are both available. If the patient is sweating or the site is bleeding or oozing, gauze dressing should be used until this is resolved.

Topical antibiotics should not be used on the insertion site because they have the potential to promote fungal infection and antibiotic resistance. Regarding gauze dressings used on short-term CVC sites, it is recommended to change them every 2 days. It is recommended that transparent dressing used on short-term CVC sites be changed every 7 days, except when infants are more likely to lose their catheter while replacing dressings. ${ }^{14)}$

\section{Chlorhexidine-impregnated dressings}

Chlorhexidine-impregnated dressings are not recommended for the protection of the site of short-term, nontunneled CVCs for premature neonates due to the risk of serious adverse skin reactions. No recommendation is made about the use of chlorhexidine-impregnated dressings to protect the site of short-term, nontunneled CVCs for pediatric patients $<18$ years old and nonpremature neonates due to the lack of sufficient evidence in this age group..$^{14,28,29)}$

\section{Umbilical catheters}

Umbilical arterial (UA) or umbilical venous (UV) catheter is commonly used in the management of sick neonates, but they can lead to serious complications including infection. To prevent catheterrelated infection, before inserting an umbilical catheter, it is recommended to disinfect the insertion site with antiseptics (tincture of iodine is contraindicated, but other iodine agents [e.g., povidone iodine] may be used). Topical antibiotics at the site of insertion are also contraindicated in the umbilical catheter. It is recommended that low-doses of heparin $(0.25-1.0 \mathrm{U} / \mathrm{mL})$ be added to the fluid injected into the UA catheter to maintain its patency. If UA and UV catheters are no longer needed, they should be removed as soon as possible. An umbilical catheter can be replaced if it malfunctions and there is no other indication for removal. The total period of catheter use should not exceed 5 days for UA catheter, or 14 days for UV catheter. When CRBSIs occur or signs of vascular insufficiency or thrombosis are seen, UA or UV catheters should be removed and never be replaced. ${ }^{14)}$

\section{Systemic prophylactic antimicrobials}

In a meta-analysis on the use of prophylactic systemic antibiotics in neonates with CVCs, its use was not effective in reducing overall mortality. In addition, there is a lack of evidence on long-term neurodevelopmental outcome and the use of antibiotics has concerns about the selection of resistant organisms. ${ }^{30)}$

\section{Antimicrobial locks}

There have been reports that using antibiotic lock solutions have been effective in lowering CRBSIs in neonates. There are data from each study on newborns in which fusidic acid, vancomycin, or amikacin was used. However, its use did not effectively lower mortality and there were insufficient data on long-term effects on antimicrobial resistance. Therefore, prophylactic antimicrobial lock solution can be considered in patients with long-term catheters who have a history of multiple CRBSI despite optimal maximal adherence to aseptic technique. ${ }^{31)}$

\section{Chlorhexidine bathing}

In adults, daily skin cleansing with $2 \%$ chlorhexidine is recommended to prevent CRBSIs, ${ }^{14)}$ but data on the efficacy and safety of chlorhexidine bathing in neonates and infants are very limited. Recently, there have been some reports that chlorhexidine bathing has reduced the incidence of CLABSI, but there is a lack of evidence in neonates and premature infants. ${ }^{32)}$

\section{Administration of IV fat emulsions}

Since lipids are essential for growth and development, infants 
and children require a higher proportion of dietary fat than adults. ${ }^{33)}$ However, IV fat emulsion (IVFE) has several safety issues especially when used in neonates and young infants. Because manufacturers do not produce small-volume products, using them in the original container with an infusion pump can cause fatal complications due to problems with the pump itself or mishandling. On the other hand, to infuse IVFE using a syringe pump, it needs to be repackaged into a syringe, during which microbial contamination may occur. Moreover, because IVFE has high growth potential for bacteria and fungi, its risk of microbial contamination is high. ${ }^{34)}$ There is no preferred infusion method.

There is a debate about proper IVFE hang time. While the American Association of Pediatrics recommends that a daily dose of IVFE should be infused during 18-24 hours due to infants' low-fat tolerance ${ }^{333}$ the Centers for Disease Control and Prevention recommend it should be infused within 12 hours. However, it is acceptable to administer its daily dose for up to 24 hours if it is unavoidable. ${ }^{14)}$

\section{Replacement of administration sets}

If not used to infuse blood, blood products, or IVFE, administration sets that are continuously used, including secondary sets and add-on devices, should be replaced no more frequently than at 96-hour intervals, but at least every 7 days. No recommendation is made regarding the frequency for replacing intermittently used administration sets. The tubing set used to administer blood, blood products, or IVFEs should be replaced within 24 hours of initiating the infusion. ${ }^{14)}$

\section{Conclusions}

Prevention of CLABSI is essential to improve the outcomes and prognoses of NICU patients. It is most important to regularly educate, train and give feedback to medical staffs about the various preventive measures required at each stage from before insertion to removal of the catheter. In order to improve the quality of infection control, continuous efforts are needed to develop effective and safe infection control strategies for neonates and young infants.

\section{Conflicts of interest}

No potential conflict of interest relevant to this article was reported.

\section{References}

1. Stoll BJ, Shane AL. Infections of the neonatal infant. In: Kliegman RM, Stanton BF, St Geme III JW, Schor NF, editors. Nelson textbook of pediatrics. 20th ed. ed. Philadelphia (PA): Elsevier Saunders, 2016: 909-25.

2. Goudie A, Dynan L, Brady PW, Rettiganti M. Attributable cost and length of stay for central line-associated bloodstream infections. Pediatrics 2014;133:e1525-32.

3. Bell T, O'Grady NP. Prevention of central line-associated bloodstream infections. Infect Dis Clin North Am 2017;31:551-9.

4. Centers for Disease Control and Prevention. National Healthcare Safety Network (NHSN) Patient Safety Component Manual 2017 [Internet]. Atlanta (GA): Centers for Disease Control and Prevention; 2017 [cited 2018 Sep 1]. Available from: https://www.cdc.gov/nhsn/ pdfs/validation/2017/pcsmanual_2017.pdf.

5. Nationales Referenzzentrum für Surveillance von nosokomialen Infektionen. NEO-KISS Protocol, Nosocomial infection surveillance for preterm infants with birthweight $<1500 \mathrm{~g}$ [Internet]. Berlin (Germany): Nationales Referenzzentrum für Surveillance von nosokomialen Infektionen; 2010 [cited 2018 Aug 16]. Available from: http:// www.nrz-hygiene.de/fileadmin/nrz/module/neo/NEO-KISSProtocol_ english_240210.pdf.

6. van der Zwet WC, Kaiser AM, van Elburg RM, Berkhof J, Fetter WP, Parlevliet GA, et al. Nosocomial infections in a Dutch neonatal intensive care unit: surveillance study with definitions for infection specifically adapted for neonates. J Hosp Infect 2005;61:300-11.

7. Mobley RE, Bizzarro MJ. Central line-associated bloodstream infections in the NICU: Successes and controversies in the quest for zero. Semin Perinatol 2017;41:166-74.

8. Schulman J, Stricof R, Stevens TP, Horgan M, Gase K, Holzman IR, et al. Statewide NICU central-line-associated bloodstream infection rates decline after bundles and checklists. Pediatrics 2011;127:436-44.

9. Couto RC, Carvalho EA, Pedrosa TM, Pedroso ER, Neto MC, Biscione FM. A 10-year prospective surveillance of nosocomial infections in neonatal intensive care units. Am J Infect Control 2007;35:183-9.

10. Chun P, Kong SG, Byun SY, Park SE, Lee HD. Analysis of neonatal sepsis in one neonatal intensive care unit for 6 years. Korean J Pediatr 2010;53;495-502.

11. Crnich CJ, Maki DG. The promise of novel technology for the prevention of intravascular device-related bloodstream infection. I. Pathogenesis and short-term devices. Clin Infect Dis 2002;34:1232-42.

12. Raad I, Costerton W, Sabharwal U, Sacilowski M, Anaissie E, Bodey GP. Ultrastructural analysis of indwelling vascular catheters: a quantitative relationship between luminal colonization and duration of placement. J Infect Dis 1993;168:400-7.

13. Aslam S. Effect of antibacterials on biofilms. Am J Infect Control 2008;36:S175.e9-11.

14. Centers for Disease Control and Prevention. Guidelines for the prevention of intravascular catheter-related infections, 2011 [Internet]. Atlanta (GA): Centers for Disease Control and Prevention; 2017 [cited 2018 Aug 16]. Available from: https://www.cdc.gov/infectioncontrol/ guidelines/bsi/.

15. Krein SL, Kuhn L, Ratz D, Chopra V.Use of designated nurse PICC teams and CLABSI prevention practices among U.S. Hospitals: a wurvey-based study. J Patient Saf 2015.

16. Tucker J; UK Neonatal Staffing Study Group. Patient volume, staffing, and workload in relation to risk-adjusted outcomes in a random stratified sample of UK neonatal intensive care units: a prospective evaluation. Lancet 2002;359:99-107.

17. Panagiotounakou P, Antonogeorgos G, Gounari E, Papadakis S, Labadaridis J, Gounaris AK. Peripherally inserted central venous catheters: frequency of complications in premature newborn depends on the insertion site. J Perinatol 2014;34:461-3.

18. Wrightson DD. Peripherally inserted central catheter complications in neonates with upper versus lower extremity insertion sites. Adv 
Neonatal Care 2013;13:198-204.

19. Vegunta RK, Loethen P, Wallace LJ, Albert VL, Pearl RH. Differences in the outcome of surgically placed long-term central venous catheters in neonates: neck vs groin placement. J Pediatr Surg 2005;40:4751.

20. Breschan C, Platzer M, Jost R, Schaumberger F, Stettner H, Likar R. Comparison of catheter-related infection and tip colonization between internal jugular and subclavian central venous catheters in surgical neonates. Anesthesiology 2007;107:946-53.

21. Schulman J, Stricof RL, Stevens TP, Holzman IR, Shields EP, Angert RM, et al. Development of a statewide collaborative to decrease NICU central line-associated bloodstream infections. J Perinatol 2009;29: 591-9.

22. Lai NM, Taylor JE, Tan K, Choo YM, Ahmad Kamar A, Muhamad NA. Antimicrobial dressings for the prevention of catheter-related infections in newborn infants with central venous catheters. Cochrane Database Syst Rev 2016;3:CD011082.

23. Williams FL, Watson J, Day C, Soe A, Somisetty SK, Jackson L, et al. Thyroid dysfunction in preterm neonates exposed to iodine. J Perinat Med 2017;45:135-43.

24. Smerdely P, Lim A, Boyages SC, Waite K, Wu D, Roberts V, et al. Topical iodine-containing antiseptics and neonatal hypothyroidism in very-low-birthweight infants. Lancet 1989;2:661-4.

25. Linder N, Davidovitch N, Reichman B, Kuint J, Lubin D, Meyerovitch $\mathrm{J}$, et al. Topical iodine-containing antiseptics and subclinical hypothyroidism in preterm infants. J Pediatr 1997;131:434-9.

26. Kieran EA, O'Sullivan A, Miletin J, Twomey AR, Knowles SJ, O'Donnell CPF. 2\% chlorhexidine-70\% isopropyl alcohol versus 10\% povidone-iodine for insertion site cleaning before central line inser- tion in preterm infants: a randomised trial. Arch Dis Child Fetal Neonatal Ed 2018;103:F101-6.

27. Foster JP, Richards R, Showell MG, Jones LJ. Intravenous in-line filters for preventing morbidity and mortality in neonates. Cochrane Database Syst Rev 2015;(8):CD005248.

28. Levy I, Katz J, Solter E, Samra Z, Vidne B, Birk E, et al. Chlorhexidineimpregnated dressing for prevention of colonization of central venous catheters in infants and children: a randomized controlled study. Pediatr Infect Dis J 2005;24:676-9.

29. Garland JS, Alex CP, Mueller CD, Otten D, Shivpuri C, Harris MC, et al. A randomized trial comparing povidone-iodine to a chlorhexidine gluconate-impregnated dressing for prevention of central venous catheter infections in neonates. Pediatrics 2001;107:1431-6.

30. Jardine LA, Inglis GD, Davies MW. Prophylactic systemic antibiotics to reduce morbidity and mortality in neonates with central venous catheters. Cochrane Database Syst Rev 2008;(1):CD006179.

31. Taylor JE, Tan K, Lai NM, McDonald SJ. Antibiotic lock for the prevention of catheter-related infection in neonates. Cochrane Database Syst Rev 2015;(6):CD010336.

32. Quach C, Milstone AM, Perpête C, Bonenfant M, Moore DL, Perreault T. Chlorhexidine bathing in a tertiary care neonatal intensive care unit: impact on central line-associated bloodstream infections. Infect Control Hosp Epidemiol 2014;35:158-63.

33. Kleinman RE. Pediatric nutrition handbook. 6th ed. Elk Grove (IL): American Academy of Pediatrics, 2009:529.

34. Crill CM, Hak EB, Robinson LA, Helms RA. Evaluation of microbial contamination associated with different preparation methods for neonatal intravenous fat emulsion infusion. Am J Health Syst Pharm 2010;67:914-8. 